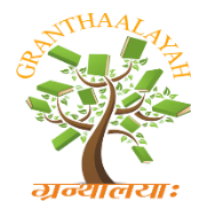

Social

\title{
DANCE AND QUALITY OF LIFE PROMOTION IN DOWN SYNDROME: A VIEW ON DEPRESSIVE AD SELF-ESTEEM ASPECTS
}

\author{
Tainah Lima Sousa Santana ${ }^{1}$, Evanilza Teixeira Adorno ${ }^{2}$, Lavinia Teixeira-Machado *3 \\ ${ }^{1}$ Nursery Department, Federal University of Sergipe, Brazil \\ ${ }^{2}$ Physical Therapy Department, Federal University of Sergipe, Brazil \\ ${ }^{* 3}$ Education in Health Department, Federal University of Sergipe, Brazil
}

\begin{abstract}
Down Syndrome (DS) adult often isolates himself from the world, due to social participation difficulty in understanding everything that surrounds him. In this context, dance assumes a substantial role in facilitating communication process, as it offers new ways of expressing ideas and feelings. The aim of this study was to investigate the effects of dance in quality of life, depressive and self-esteem aspects in DS. Method: This is a case report, longitudinal, descriptive, retrospective and prospective study, with dance class, twice a week, and public presentations, during four years. It was analyzed quality of life, depression index and self-esteem. Results: During the years, participant presented a gradual improvement in quality of life; the frequency and intensity of depression and anxiety symptoms decreased; she improved index of corporal satisfaction and in the self-esteem. Conclusion: Study reached the proposals in quality of life, depression, body satisfaction index and self-esteem.
\end{abstract}

Keywords: Down Syndrome; Dance; Quality of Life; Depression; Body Satisfaction; Self-Esteem.

Cite This Article: Tainah Lima Sousa Santana, Evanilza Teixeira Adorno, and Lavinia TeixeiraMachado. (2018). "DANCE AND QUALITY OF LIFE PROMOTION IN DOWN SYNDROME: A VIEW ON DEPRESSIVE AD SELF-ESTEEM ASPECTS." International Journal of Research - Granthaalayah, 6(5), 37-46. https://doi.org/10.29121/granthaalayah.v6.i5.2018.1419.

\section{Introduction}

Down Syndrome (DS) is characterized by the presence of an extra amount of material on chromosome 21. It is the most common chromosomal syndrome in humans and is the main cause of developmental delay and intellectual disability in this population. Differences in DS arise from individual genetic factors, from external stimuli, education, nutrition, and the family and social context; therefore, degrees can not be attributed to DS. DS may affect any ethnicity and social class. ${ }^{1}$

DS adult often isolates himself from the world, due to social participation difficulty in understanding everything that surrounds him. In this context, art assumes a substantial role in 
facilitating communication process, as it offers new ways of expressing ideas and feelings. In this way, dance presents itself as a proposal of intervention to reduce limitations, fears and longings in $\mathrm{DS}^{2}$

Dance provides educational, physical, social and psychological benefits. Physicists include: improved coordination, strength and endurance; development of motor skills, such as: balance, gait velocity and myo-articular flexibility. Social skills include communication promotion and reduction of social anxiety. In addition, dance favors self-esteem and self-confidence. ${ }^{3}$

For Mahy, ${ }^{4}$ dance presents itself as a possibility of social insertion, health promotion and improvement of quality of life for adults with DS, making them more independent, and thus allows interaction and interpersonal relationship, especially for confronting attitudinal barriers and breaking prejudices.

The act of dancing in DS population helps the process of motor learning, social relationship, cognitive and affective skills. Dancing decreases risk factors, such as cardiovascular and musculoskeletal complications, as well as avoiding psychological disorders, such as depression and anxiety. In addition, it influences mood and decreases depression and stress, signs present in DS little evoked in the evidence point of view. ${ }^{5}$

World Health Organization (WHO) defined Quality of Life (QOL) as individual's perception of his/her position in life in the context of culture, and the system of values in which his/her lives, as well as his/her goals, expectations, standards and concerns. QOL is subjective, multidimensional and includes evaluation elements, both positive and negative. ${ }^{6}$

In addiction to health aspects, QOL involves physical, mental and environmental well-being, besides other aspects of life, as known: Family, friends and job. Because of this, it is important analyze QOL in DS due to difficult and barriers for this population. ${ }^{7}$

DS adulthood have great chances to develop depression due to social exclusion. ${ }^{8}$ Dance allows expressiveness, for the purpose to expose emotions and show that limitations can be supplanted; by dance, DS can manifest different lived experiences and succeed other way of communication between he or herself and the world around. ${ }^{9}$ Thus, this study hypothesized in first plan that dance influences DS QOL, and in second plan, dance interferes in depression index, corporal satisfaction and self-esteem in DS.

\section{Method}

\section{Study Design}

This interventional, descriptive, longitudinal, prospective case report was realized during four years. Case report was chosen, because it allows researchers focus in one case, and retain information through holistic and real world perspectives, it contributes to investigate a empirical topic following set of desired procedures. ${ }^{10}$

\section{Sample}


When participant was allocated in TALT program, she was 38 years-old, with depression (using paroxetine $20 \mathrm{mg} /$ day), dismay complaints, lack of interest in daily living tasks, relate to others, unwilling to food and to self-care.

\section{Ethical Considerations}

The study was approved by Ethical Committee in Humans (CAAE 06154012.4.0000.0058), and data collection was started after informed consent signature by participant's sponsor.

\section{Dance Protocol}

Participant attended a special dance program developed for people with disabilities (called TALT) in a suitable place, under supervision of a trained dancing master. Participant underwent dance classes twice a week, lasting $60 \mathrm{~min}$, during four years. Dancing class protocol was composed by four sets: (1) consciousness of global movements: recognizing the whole body; (2) motor coordination: rhythmic activities on the ground, joining simultaneous components of movement; (3) sensorial integration: subject-environment interaction, and internal perception of movement; (4) skill and agility: space-temporal orientation, balance and performance learning. Besides, performances were exposed on the stage, schools, universities and other events.

\section{Data Collection and Analysis}

Data were collected during four years through measurements that reflect study proposal. Trained examiners applied measurements in five moments: baseline, and after 12 months of intervention. Follow up period was 15 days.

\section{Measurements}

\section{Quality of Life Questionnaire Short-form Survey SF-36 (SF-36)}

SF-36 is gold pattern to measure QOL by participant point of view. It has easy administration and comprehension, multidimensional, formed by 36 items, and eight domains: functional capacity, physical aspects, pain, general state of health, vitality, social aspects, mental health and emotional aspects. Score range between 0-100, being 0 worst QOL and 100 better state of QOL. ${ }^{11,12}$

\section{Beck Depression Inventory (BDI)}

This inventory is widely used to measure the severity of depression. It consists of 21 multiplechoice self-reporting questions. Its development marked a change among health professionals, which until then was seen depression like a psychodynamic perspective, rather than being visualized by the patient himself. BDI consists of 21 questions about how the subject feels in past week. Each question has four alternatives in which only one should be marked, punctuating the intensity of the sensation. For example: (0) I do not feel sad; (1) I feel sad; (2) I am sad all the time and I can not get out of it; (3) I am very sad or unhappy that I can not bear. The score varies from 0 to 63 points, the higher the value, the greater the depressive state. ${ }^{13,14}$

\section{Body Shape Questionnaire (BSQ)}

The BSQ assesses the level of body image dissatisfaction in youth and adults; it allows us to distinguish two specific aspects of body image: the accuracy of body size estimation and feelings about body, dissatisfaction or depreciation. A score of less than or equal to 80 points is found normality pattern and considered as absence of distortion of body image. Results between 81 and 
110 points are classified as slight distortion of body image. Data obtained between 111 and 140 points are classified as moderate distortion of body image. And above 140 points, the classification is represented as severe distortion of body image. ${ }^{15,16}$

\section{Rosenberg Self-esteem Scale (RSES)}

Self-esteem can be measured by RSES, a one-dimensional instrument capable of classifying low, medium, and high self-esteem. It has ten items, five of which refer to positive "self-image" or "self-value" and five to "negative self-image" or "self-depreciation". Response options are arranged in four points, ranging from "totally agree" to "totally disagree". RSES allows a total result, ranging from 10 to 40, increasing as a function of the level of self-esteem. Some studies point to self-esteem as an important indicator of mental health, besides being an important tool to identify and prevent psychological problems; Thus, it is fundamental to study improvements in self-esteem that dance can provide in DS. ${ }^{17,18}$

\section{Results and Discussions}

Assess measures were applied before starting dance classes (baseline) and every twelve months of intervention. Follow up corresponds to interval between the years (period of celebrations of the end of each year and beginning of the following year - that was equivalent to about fifteen days) (Figure 1).

Figure 2 shows obtained data for each SF-36 Questionnaire domain during 4 years of dance intervention. From the analysis of the domains, there are changes over the years, gradually increasing quality of life of the participant, mainly in domains: social, physical and vitality aspects. Emotional aspects and pain reached $100 \%$.

In relation to depression index verified by BDI, it was observed a reduction of intensity and frequency of the symptoms of depression and anxiety after dance practice over the years. The score of first evaluation was 36, in second year, 15 in third year, 12 points, and in forth year the score was 5 (Figure 3).

Figure 1 - Timeline of the study.
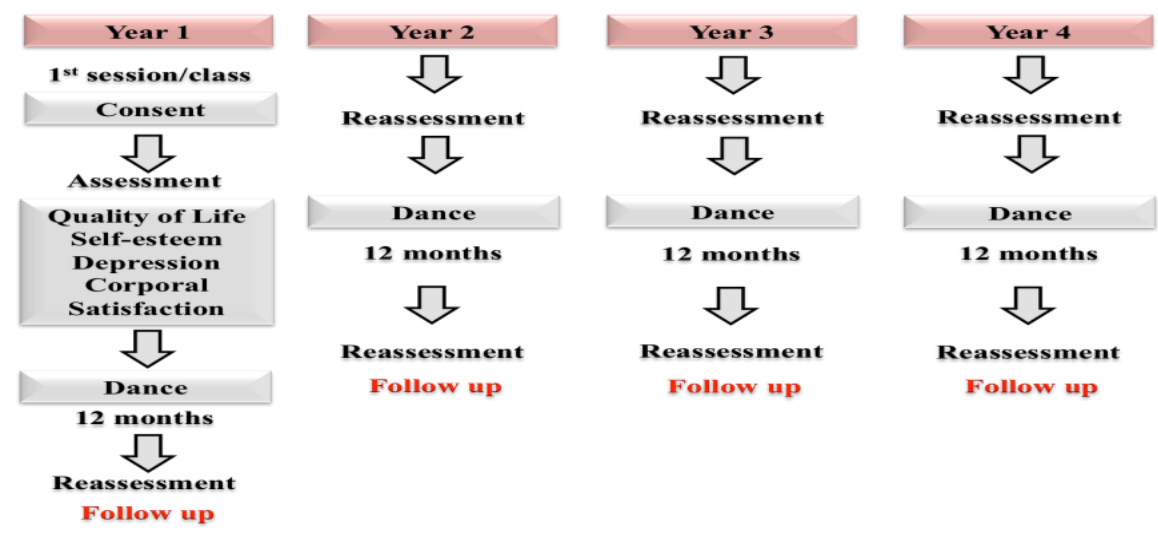

Follow up

Source: TALT Group 
Figure 2 - SF-36 Quality of Life survey values during four years, before and after dance intervention. Domains: Functional capacity, physical aspects, social aspects, emotional aspects, vitality, general state of health, pain, and mental health.

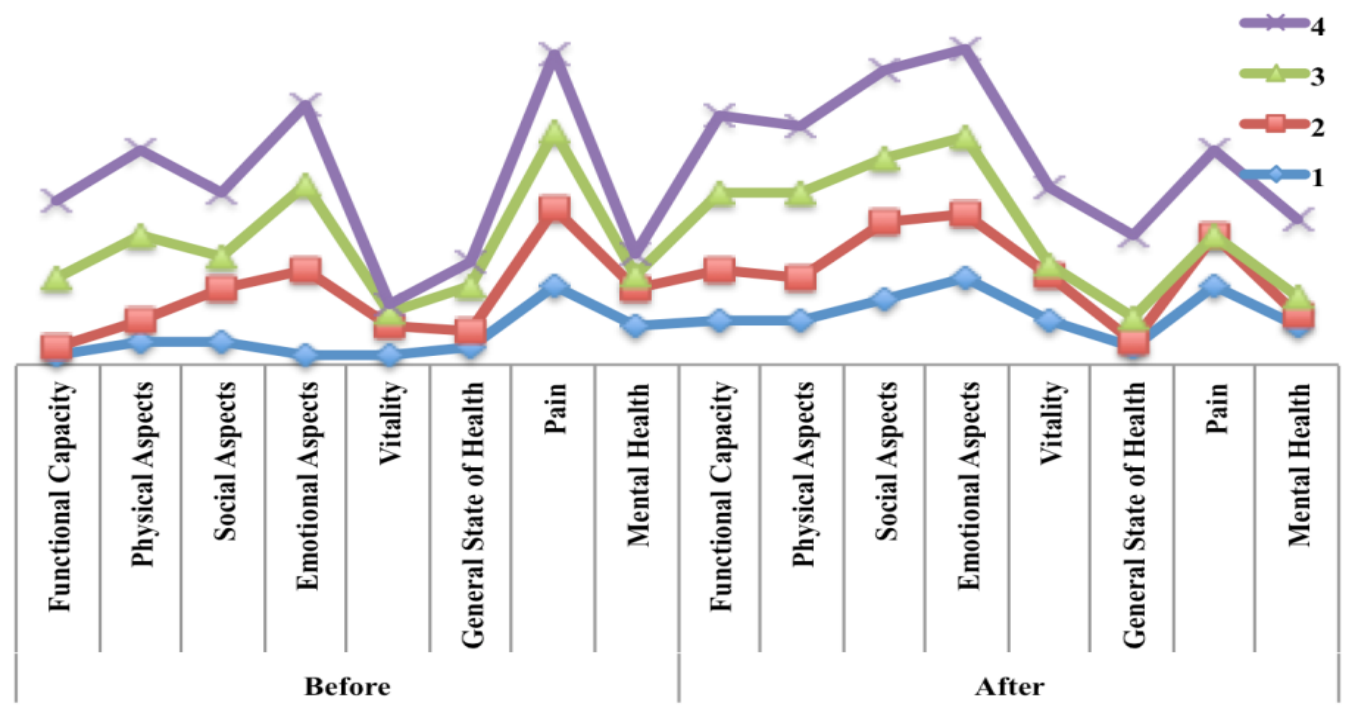

Source: TALT

Current BDI version is intended for individuals with 13 years or older and is composed of items related to symptoms of depression, such as lack of hope and irritability, perceptions such as feelings of guilt or punishment, physical symptoms such as fatigue, weight loss and lack of interest in sex. It is worth mentioning that the BDI maximum score is 40 points that indicates severe depression $^{13}$.

Figure 4 shows body satisfaction values before and after dance practice over the four years. When the participant arrived at project, she presented serious alteration in relation to her body perception (170 points), in specific aspects of body image, regarding feelings of dissatisfaction or devaluation of herself body. In subsequent years, there was a reduction in body satisfaction index, and in final year, score was 99.

Figure 3 - Beck Depression Inventory values before and after every year of dance intervention.

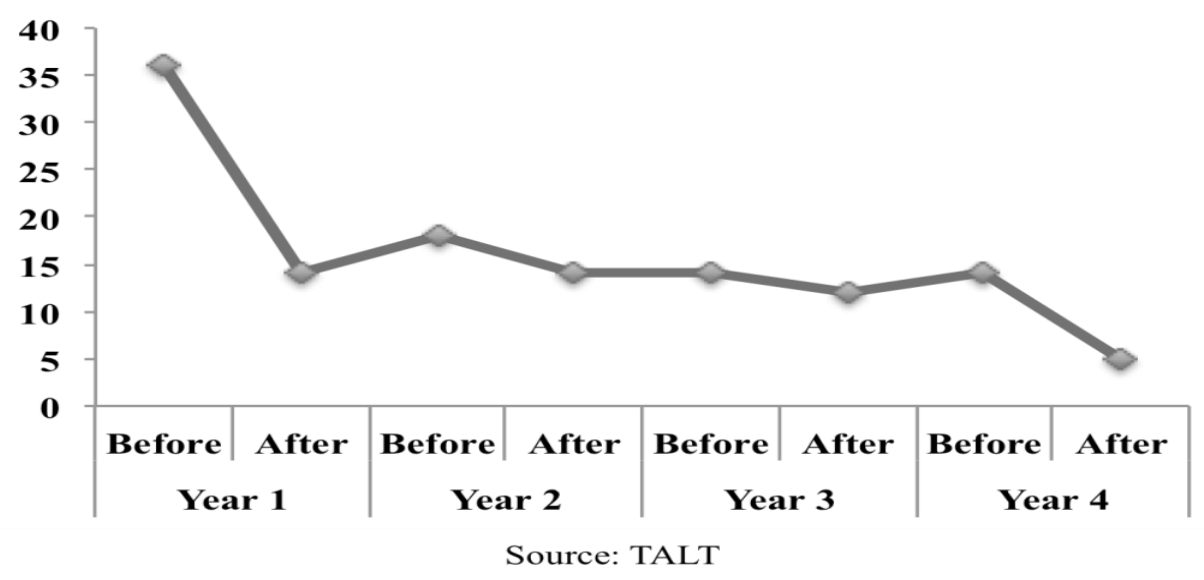


Figure 5 presents values obtained in relation to the self-esteem over the 4 years of the participant's dance. In first year, score was 10 at the beginning of the study, at the end was 14 points; in second year, she presented 17 points in RSES; in third year, 14 points at the beginning and 17 at the end; and in forth year, 17 points at the beginning and 24 at the end of intervention.

Researches show how favorable dance is in the development of who practices it, but unfortunately studies with DS people are scarce. According to the Arts Council of England (2006) "anyone can practice dance regardless of his/her age, whether or not they are disabled, whether or not have practiced dance, whatever shape and size".

Figure 4 - Corporal satisfaction values by Body Shape Questionnaire (BSQ), before and after dance intervention during four years.
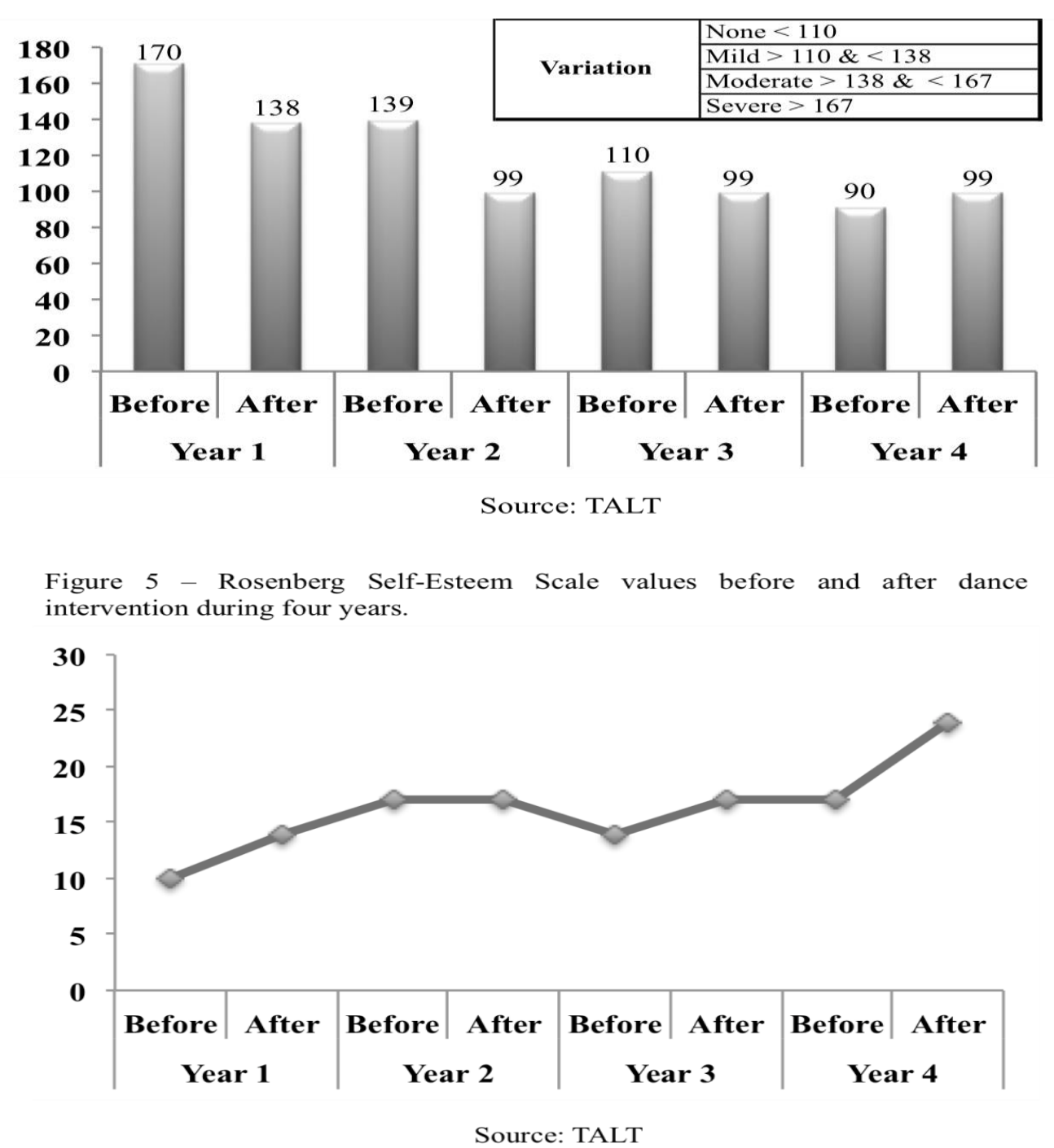

Dance arouses factors associated with body abilities, from emotions revealed by corporal movement, providing coordination, strength, endurance and motor skills, such as: gait speed, balance and flexibility; aspects that influence, substantially, corporal perception, space-temporal orientation, communication; in addition to reducing anxiety and increasing self-esteem, selfconfidence and quality of life. ${ }^{19}$ 
Findings demonstrated the influence of dance on the participant's QOL, because through the results of the SF-36 it was possible to perceive changes in all domains that are directly related to the current concept of QOL, as well as in the study by Teixeira-Machado and De Santana ${ }^{12}$ that showed significant changes in QOL through dance in functional capacity, social and emotional aspects domains.

The present study showed the importance of dance in DS, reinforcing what Reinders, Bryden \& Flectcher ${ }^{19}$ demonstrated in their study in functional and psychosocial aspects, including affectivity. During dance classes, in addition to motor and functional improvement, it was noticed development in social aspects, emphasizing communication and social interaction.

Psychosocial aspects deserve special mention, especially when it comes to verbal and non-verbal communication, besides memory. Communication and relationship have become easier in course of dance classes; this was evidenced in the understanding and learning of choreographic sequences.

Another major issue was the awakening of the ability to convey desires through non-verbal communication. Most of the studies with a similar intervention proposal addresses dance benefits in the emotions transcendence, leading to momentary loss of consciousness and space, which creates an absorption of the moment that leads to internal and external improvements for the one who dances. $5,20,21$

In addition, participant's sister referred improvements after dance practice: before participant had a severe depressive condition, with accompaniment of psychiatrists and psychologists, use of medication to sleep, "she lived sad and isolated", with difficulties of socialization. Currently, she says that she loves dancing and participating in the dance program, which was "the realization of a dream to become a dancer". In addition, she has improved family life, participates in social networks, church groups, works in the family business, and has suspended the use of sleeping medication.

Hedov et $\mathrm{al}^{11}$ and Chen \& Ringenbach ${ }^{21}$ also showed QOL improvements in individuals with DS who practice some physical activity; and they also reported an increase in life expectancy in this group, probably due to sociocultural activities, as perceived in the present study.

Ito et $\mathrm{al}^{5}$ and Ptomey et $\mathrm{al}^{2}$ affirmed that dance helps the reduction of risks in locomotive apparatus, as well as depression and anxiety; in addition to reaffirming the effects of dance in the mood, depression and daily stress, in social reciprocity, and, above all, reduction of the continuous use of drugs.

Body satisfaction findings (Figure 4) reaffirm Gutiérrez-Vilahú et $\mathrm{al}^{22}$ study on the influence of dance on physical and intellectual activity in DS. Living, according to O'Brien, ${ }^{23}$ does not disassociate from the relationship with herself and the others, and this reflects in the subjectivation of body perception and satisfaction, how it is seen by her and how it is seen by others.

Besides, DS predisposes overweight or obesity due to slow metabolic rate, abnormal concentrations of leptin in the blood and low levels of physical activity. Deficiencies of vitamin B 
groups and abnormal levels of homocysteine in the blood also decrease the rate of intellectual development in DS. . $^{1,21,24}$

So, according to findings, dance is a physical activity and presents itself as an intervention proposal that assists in maintaining body weight in DS. Practice of this art promotes bodily satisfaction and appreciation of how one is, not according to proposed standards.

Figures 4 and 5 show improvements as a result of dance practice, as well as public presentations, both in body satisfaction and in self-esteem. The scale used to monitor self-esteem presents points directly related to obesity.

DeFarias \& Teixeira-Machado ${ }^{9}$ and Jung et $\mathrm{al}^{25}$ point out that DS QOL has been changing over the last few years, both due to the change in life expectancy in these individuals and the increase in proposals for interventions that interfere in QOL, such as dance. Moreover, these findings reaffirm the dance influence in social aspects and mental health, verified in the results about QOL, depression and self-esteem.

Marques and Nahas $^{26}$ explained that the practice of activities such as dance improves communication and socialization in DS, especially in their daily relationships, creating possibilities for social interaction, personal satisfaction and self-esteem, fundamental for life in this population.

The results of this study reaffirm those of $\mathrm{Mahy}^{4}$ and Mala, Karkou and Meekums, ${ }^{27}$ in elucidating that dance represents a possibility of social insertion and improvement of DS QOL. Even with regard to symptoms of depression and anxiety, which greatly influence QOL, without refuting the importance of self-esteem and lifestyle.

For Teixeira-Machado\&DeSantana ${ }^{12}$ and Hardee\&Fetters ${ }^{28}$ dance provides benefits related to body awareness, which favors substantially self-image construction. In addition, it promotes social and emotional integration, and thus allows for self-acceptance and social reciprocity happens.

Dance promotes body expression by stimulating brain regions that involve attention, prediction, and intent of cognitive, creative, and artistic skills that compose the personal and social framework of the dance practitioner. ${ }^{19}$

\section{Conclusions and Recommendations}

Findings showed that dance influenced quality of life domains, mainly emotional, physical, social, vitality and mental health aspects. In relation to depression, body satisfaction and self-esteem, dance practice highlighted the possibility of developing independence, autonomy and fostering the expression of being in its totality.

Dance practice interfered in cognitive processes for movement elaboration by inquiring sensorial stimuli that favored the amplification of the functional capacity. 
Using dance as a tool to improving neuromotor apparatus with the aim of favoring satisfaction with body image and self-esteem has shown to be important in emotional aspects, because it embodies meaning and motivation to participate in an activity that promotes physical and emotional well-being.

In relation to social aspects, dance practice along the years enabled the insertion in social contexts by providing participation in socio-cultural activities.

\section{References}

[1] Mazurek D, Wyka J. Down syndrome - genetic and nutritional aspects of accompanying disorders. Rocz Panstw Zakl Hig, 2015;66(3):189-94.

[2] Ptomey LT, Szabo AN, Willis EA, Gorczyca AM, Greene JL, Danon JC, Donnelly JE. Changes in cognitive function after a 12-week exercise intervention in adults with Down syndrome. Disabil Health J, 2018, pii: S1936-6574(18)30038-4. doi: 10.1016/j.dhjo.2018.02.003.

[3] Becker E, Drusing S. "Participation is Possible: A Case Report if Integration into a Community Performing Arts Program.” Physiotherapy Theory and Practice v.26, n.4, 2010.

[4] Mahy JN, Shields NF, Taylor KJ. Identifying Facilitators and Barriers to Physical Activity for Adults with Down Syndrome. Journal of Intellectual Disability Research, 2010, 54(9):795-805.

[5] Ito Y, Hiramoto I, Kodama H. Factors affecting dance exercise performance in students at a special needs school. Pediatr Int, 2017, 59(9):967-972.

[6] Barbosa RTA, de Oliveira ASB, de Lima Antão JYF, Crocetta TB, Guarnieri R, Antunes TPC, Arab C, Massetti T, Bezerra IMP, de Mello Monteiro CB, de Abreu LC. Augmentative and alternative communication in children with Down's syndrome: a systematic review. BMC Pediatr, 2018, 18(1):160.

[7] Newton R. Quality of life in Down syndrome: a matter of perspective. Dev Med Child Neurol. 2018, 60(4):337-338.

[8] Tekinarslan IC. A comparison study of depression and quality od life in Turkish mothers of children with Down syndrome, cerebral palsy, and autism spectrum disorder. Psychol Rep, 2013, 112(1):266-287.

[9] DeFarias LHS, Teixeira-Machado L. Behind the Dance: Educational, Emotional and Social Contexts in Down syndrome. International Journal of Humanities Social Sciences and Education (IJHSSE), 2016, 3(1):20-23.

[10] Feldman M, McDonald L, Serbin L, Stack D, Secco ML, Yu CT. Predictors of depressive symptoms in primary caregivers of young children with or at risk for developmental delay. $\mathrm{J}$ Intellect Disabil Res. 2007 Aug;51(Pt 8):606-19.

[11] Hedov G, Annéren G, Wikblad k. Self-perceived health in Swedish parents of children with Down's syndrome. Qual Life Res, 2000, 9(4):415-422.

[12] Teixeira-Machado L, DeSantana JM. Dance Therapy Improves Quality of Life in Individuals with Neuromotor Disorders: Randomized Controlled Trial. International Journal of Humanities Social Sciences and Education, 2015, 2(4):84-92.

[13] Beck AT, Ward CH, Mendelson M, Mock J, Erbaugh J. An inventory for measuring depression. Arch. Gen. Psychiatry, 1961, 4:561-71.

[14] Li EPY, Liu YM, Lok NCY, Lee VWK. Successful experience of people with Down syndrome. J Intellectual Disabilities, 2006, 10(2).

[15] Radwan H, Hasan HA, Najm L, Zaurub S, Jami F, Javadi F, Deeb LA, Iskandarani A. Eating disorders and body image concerns as influenced by family and media among university students in Sharjah, UAE. Asia Pac J Clin Nutr. 2018;27(3):695-700.

[16] Miyamoto ST, Lombardi Junior I, Berg KO, Ramos LR, Natour J. Brazilian version of the Berg balance scale Braz J Med Biol, 2004, 37(9):1411-1421. 
[17] Rosenberg M. Society and the adolescent self-image. Princeton, NJ: Princeton University Press, 1965.

[18] Sbicigo JB, Bandeira DR, Dell'Aglio DD. Escala de Autoestima de Rosenberg (EAR): validade fatorial e consistência interna. Psico-USF, 2010, 15(3):395-403.

[19] Reinders N, Bryden PJ, Fletecher PC. Dancing with Down syndrome: a phenomenological case study. Research in Dance Education, 2015.

[20] Alonso DA, Marcos FML, Miguel PAS, Oliva DS, Calvo TG. Interacion de la teoria de la autodeterminacion em la fluidez disposicional em praticantes de danza. Cuaderno de Psicologia del puerto, 2011, 11(1):7-17.

[21] Chen CJJ, Ringenbach SDR. Walking performance in adolescents and young adults with Down syndrome: the role of obesity and sleep problems. J Intellect Disabil Res. 2018 Apr;62(4):339-348.

[22] Gutiérrez-Vilahú L, Massó-Ortigosa N, Costa-Tutusaus L, Guerra-Balic M, Rey-Abella F. Comparison of static balance on a plataform in young adults with Down syndrome before and after a dance program. Adapt Phys Activ Q. 2016 Jul; 33(3):233-52.

[23] O'Brien PC. Performance government: activating and regulating the self-governing capacities of teachers and school leaders. Educational Philosophy and Theory, 2015, 47(8):833:847.

[24] Gonçalves Machado A, Lummertz Magenis M, Bongiolo AM, Castro K, da Silva MA, Schweigert Perry ID. Bioeletrical impedance analysis: body composition in children and adolescents with Down syndrome. Minerva Pediatr. 2017 Dec; 69(6):560-563.

[25] Jung HK, Chung E, Lee BH. A comparison of the function, activity and participation and quality of life between Down syndrome children and typically developing children. J Phys Ther Sci. 2017 Aug; 29(8):1377-1380.

[26] Marques AC, Nahas MV. Qualidade de vida de pessoas portadoras de Síndrome de Down, com mais de 40 anos, no Estado de Santa Catarina. Revista Brasileira de Ciência e Movimento, 2003, 11(2):55-61.

[27] Mala A, Karkou V, Meekums B. Dance/Movement therapy for depression: a scoping review. The arts in Psychotherapy, 2012, 39(4):287-295.

[28] Hardee JP, Fetters L. The effect of exercise intervention on daily life activities and social participation in individuals with Down syndrome: a systematic review. Research in Developmental Disabilities, 2017, 62:81-103.

*Corresponding author.

E-mail address: teixeiramachado@ icloud.com 